\title{
LINEAR EQUATIONS WITH ONE CONSTRAINT AND THEIR CONNECTION TO NONLINEAR EQUATIONS OF THE FOURTH ORDER
}

\author{
ALEXANDER CHICHURIN AND GALINA FILIPUK
}

Received 04 May, 2019

\begin{abstract}
The purpose of this paper is to present several new results concerning relations between linear differential equations of the fourth order with one constraint and nonlinear differential equations of the fourth order. We consider linear differential equations of the second, the third and the fourth order and nonlinear fourth order differential equations related via the Schwarzian derivative. The method is based on the use of the Schwarzian derivative, which is defined as the ratio of two linearly independent solutions of the linear differential equations of the second or third and fourth order. As a result we obtain new relations between the solutions of these linear and nonlinear equations. To illustrate theorems and our constructive approach we give two examples. The given method may be generalized to differential equations of higher orders.
\end{abstract}

2010 Mathematics Subject Classification: 34A05; 34A30; 34A34

Keywords: Schwarzian derivative, fourth order nonlinear differential equation, properties linear differential equation of the fourth order

\section{INTRODUCTION}

The Schwarzian derivative is a differential operator that is invariant under all linear fractional transformations, see $[1,8,10]$. It plays a significant role in the theory of modular forms, hypergeometric functions, univalent functions and conformal mappings $[1,8]$. It is defined by

$$
(S f)(z)=\left(\frac{f^{\prime \prime}(z)}{f^{\prime}(z)}\right)^{\prime}-\frac{1}{2}\left(\frac{f^{\prime \prime}(z)}{f^{\prime}(z)}\right)^{2}=\frac{f^{\prime \prime \prime}(z)}{f^{\prime}(z)}-\frac{3}{2}\left(\frac{f^{\prime \prime}(z)}{f^{\prime}(z)}\right)^{2} .
$$

The well-known relation between a second-order linear differential equation of the form

$$
y^{\prime \prime}(z)+Q(z) y(z)=0
$$

The second author acknowledges the support of the Alexander von Humboldt Foundation and the support of National Science Center (Narodowe Centrum Nauki NCN) OPUS grant 2017/25/B/BST1/00931 (Poland). 
and the Schwarzian derivative of the ratio of two linearly independent solutions $y_{1}$, $y_{2}$ of the linear equation above is as follows:

$$
(S \xi)(z)=2 Q(z)
$$

where $\xi=y_{1} / y_{2}$ and $z$ is, in general, a complex variable. See [1] and [8] for more details.

If we have a general second order differential equation

$$
y^{\prime \prime}(z)+p(z) y^{\prime}(z)+q(z) y(z)=0,
$$

then substituting $y(z)=\xi(z) y_{1}(z)$ with the condition that $y_{1}$ is also a solution of (1.1), we get an expression for $\xi, y_{1}$ and their derivatives (up to order 2 and 1 respectively). Differentiating again and eliminating $y_{1}, y_{1}^{\prime}$, we get that the function

$$
w(z)=(S \xi)(z)
$$

satisfies

$$
w(z)=\frac{1}{2}\left(4 q(z)-p(z)^{2}-2 p^{\prime}(z)\right) .
$$

We call expression (1.2) the invariant for the second order linear differential equation (1.1).

On extension of this approach for linear differential equation of the third order see in [10-12]. The generalization of the method for the linear differential equations of the fourth order is given in [6]. In papers [2,4,5] special classes of the fourth order linear differential equations and the nonlinear fourth order differential equations related via the Schwarzian derivative are considered and general solutions of both differential equations are found. In paper [3] the generalization of the method for a special type of linear differential equations of the fifth order is given along with a computer realization of this method in Mathematica (www.wolfram.com).

Several questions arise. What happens if the second, the third and the fourth order linear differential equations are related? What happens if we modify the function to be the ratio of solutions of two different equations? These questions were answered during the studies of linear differential equation of the third order in [9].

Similar questions were resolved for the fours order ordinary differential equations with coefficients satisfying a system of two first order differential equations [7].

The main objective of this paper is to answer these questions for linear differential equation of the fourth order with coefficients that satisfy the differential equation of the first order. The proofs of statements are computational, that is the results can be verified by using any computer algebra system.

\section{MAIN RESUltS}

In this section we shall present 5 main results concerning the relations between linear

$$
y^{\prime \prime \prime \prime}(z)+p(z) y^{\prime \prime \prime}(z)+q(z) y^{\prime \prime}(z)+r(z) y^{\prime}(z)+s(z) y(z)=0,
$$


where

$$
p^{\prime}=\frac{1}{12}\left(8 q-3 p^{2}\right)
$$

and nonlinear differential equations.

Theorem 1. Let $y$ be a solution of the fourth order linear differential equation (2.1) and $y_{1}$ be a solution of another fourth order linear differential equation

$$
y^{\prime \prime \prime \prime}(z)+p_{1}(z) y^{\prime \prime \prime}(z)+q_{1}(z) y^{\prime \prime}(z)+r_{1}(z) y^{\prime}(z)+s_{1}(z) y(z)=0,
$$

where

$$
p_{1}^{\prime}=\frac{1}{12}\left(8 q_{1}-3 p_{1}^{2}\right) .
$$

If the function $w(z)=(S \xi)(z)$ with $\xi=y / y_{1}$ solves a nonlinear differential equation

$$
\sum_{k=0}^{1} \sum_{j=0}^{2} \sum_{i=0}^{3} \alpha_{i j k}(z) w^{i}\left(w^{\prime}\right)^{j}\left(w^{\prime \prime}\right)^{k} w^{\prime \prime \prime \prime}+\sum_{l=0}^{2} \sum_{k=0}^{3} \sum_{j=0}^{4} \sum_{i=0}^{6} \beta_{i j k l}(z) w^{i}\left(w^{\prime}\right)^{j}\left(w^{\prime \prime}\right)^{k}\left(w^{\prime \prime \prime}\right)^{l}=0,
$$

which is explicitly given by

$$
\begin{aligned}
\left(240 w w^{\prime \prime}-300 w^{\prime 2}-40 \psi w^{\prime}+160 w^{3}+\left(80 q^{\prime \prime}+\frac{40}{3} q^{2}-10 p^{2} q+60 p r-480 s-20 \phi\right) w\right. \\
\left.-\psi^{2}\right) w^{\prime \prime \prime \prime}-280 w w^{\prime \prime \prime} 2+\left(\left(56 \psi+840 w^{\prime}\right) w^{\prime \prime}-\left(1120 w^{2}-110 \phi+55 p \psi-20 \psi^{\prime}\right) w^{\prime}\right. \\
-\frac{448}{3} \psi w^{2}+\left(10 p q^{2}-\frac{5}{2} p^{3} q-\frac{45}{4} p^{2} \psi-90 \phi^{\prime}+45 p \psi^{\prime}-\frac{20}{3} q\left(3 r-4 \psi-3 p^{\prime \prime}\right)-20 \psi^{\prime \prime}\right) w \\
\left.+4 \phi \psi-2 p \psi^{2}+2 \psi \psi^{\prime}\right) w^{\prime \prime \prime}-504 w^{\prime \prime 3}+\left(192 w^{2}-153 \phi+\frac{153}{2} p \psi-66 \psi^{\prime}\right) w^{\prime \prime 2} \\
+\left(2040 w w^{\prime 2}+448 w^{4}-7 \phi^{2}-\frac{5}{16} p^{2} \psi^{2}-\frac{23}{6} q \psi^{2}+\frac{23}{2} \psi \phi^{\prime}+\phi\left(7 p \psi-18 \psi^{\prime}\right)\right. \\
+\frac{13}{4} p \psi \psi^{\prime}-2 \psi^{\prime 2}+\left(42 p \psi-84 \phi+152 \psi^{\prime}\right) w^{2} \\
+\left(\frac{55}{3} \psi^{2}-\frac{15}{4} p^{3} \psi-30 r \psi+15 p^{2} \psi^{\prime}-40 q \psi^{\prime}+60 \phi^{\prime \prime}+15 p\left(q \psi-2 \psi^{\prime \prime}\right)\right) w \\
\left.+\psi \psi^{\prime \prime}+\left(\frac{135}{8} p^{2} \psi-45 q \psi+528 \psi w+135 \phi^{\prime}-\frac{135}{2} p \psi^{\prime}+30 \psi^{\prime \prime}\right) w^{\prime}\right) w^{\prime \prime} \\
-1275 w^{\prime 4}-520 \psi w^{\prime 3}+\left(-560 w^{3}+\frac{15}{2}\left(38 \phi-19 p \psi-4 \psi^{\prime}\right) w+\frac{75}{16} p^{3} \psi\right. \\
\left.+\frac{75}{2} r \psi-\frac{611}{12} \psi^{2}-\frac{75}{4} p^{2} \psi^{\prime}+50 q \psi^{\prime}-75 \phi^{\prime \prime}-\frac{75}{4} p\left(q \psi-2 \psi^{\prime \prime}\right)\right) w^{\prime 2} \\
+\left(-128 \psi w^{3}+\frac{35}{32} p^{2} \phi \psi-\frac{35}{12} q \phi \psi+\frac{5}{64} p^{3} \psi^{2}-\frac{25}{24} p q \psi^{2}+5 r \psi^{2}-\frac{7}{6} \psi^{3}\right. \\
+\frac{35}{4} \phi \phi^{\prime}+\frac{5}{16} p^{2} \psi \psi^{\prime}+5 q \psi \psi^{\prime}+5 \phi^{\prime} \psi^{\prime}+\left(32 \phi \psi-16 p \psi^{2}+16 \psi \psi^{\prime}\right) w \\
-10 \psi \phi^{\prime \prime}-\frac{5}{8} p\left(\psi^{\prime}\left(7 \phi+4 \psi^{\prime}\right)+\psi\left(7 \phi^{\prime}-2 \psi^{\prime \prime}\right)\right)
\end{aligned}
$$




$$
\begin{aligned}
& \left.-\left(\frac{45}{2} p^{2} \psi-60 q \psi+180 \phi^{\prime}-90 p \psi^{\prime}+40 \psi^{\prime \prime}\right) w^{2}+\frac{15}{2} \phi \psi^{\prime \prime}\right) w^{\prime}+64 w^{6} \\
& -\left(8 \phi-4 p \psi-\frac{272}{3} \psi^{\prime}\right) w^{4}-\left(\frac{5}{2} p^{3} \psi+20 r \psi+\frac{82}{9} \psi^{2}-10 p^{2} \psi^{\prime}+\frac{80}{3} q \psi^{\prime}-40 \phi^{\prime \prime}\right. \\
& \left.-10 p\left(q \psi-2 \psi^{\prime \prime}\right)\right) w^{3}+\left(344 p \psi \psi^{\prime}-84 \phi^{2}-105 p^{2} \psi^{2}+4 \phi\left(21 p \psi-4 \psi^{\prime}\right)\right. \\
& \left.+16\left(14 q \psi^{2}-42 \psi \phi^{\prime}+31 \psi^{\prime 2}-28 \psi \psi^{\prime \prime}\right)\right) \frac{w^{2}}{48}+\left(\frac{13}{24} \phi \psi^{2}-\frac{5}{32} p^{3} \phi \psi-\frac{5}{9} q^{2} \psi^{2}\right. \\
& +\frac{10}{3} q \psi \phi^{\prime}-5 \phi^{\prime 2}-\frac{5}{3} q \phi \psi^{\prime}+\frac{5}{12} \psi^{2} \psi^{\prime}-\frac{10}{3} q \psi^{\prime 2}-\frac{5}{4} r \psi\left(\phi+2 \psi^{\prime}\right) \\
& +\frac{5}{48} p^{2}\left(q \psi^{2}-12 \psi \phi^{\prime}+6 \phi \psi^{\prime}\right)+\frac{5}{2} \phi \phi^{\prime \prime}+5 \psi^{\prime} \phi^{\prime \prime}+\frac{5}{3} q \psi \psi^{\prime \prime}-5 \phi^{\prime} \psi^{\prime \prime} \\
& \left.+\frac{1}{48} p\left(30 r \psi^{2}-13 \psi^{3}+240 \phi^{\prime} \psi^{\prime}+10 q \psi\left(3 \phi+2 \psi^{\prime}\right)-60 \psi \phi^{\prime \prime}-60 \phi \psi^{\prime \prime}\right)\right) w \\
& +\frac{1}{32}\left(3 \phi^{2}\left(p \psi-4 \psi^{\prime}\right)-2 \phi^{3}+2 \psi\left(2 r \psi^{2}-3 p \psi \phi^{\prime}+8 \phi^{\prime} \psi^{\prime}-4 \psi \phi^{\prime \prime}\right)\right. \\
& \left.+2 \phi\left(\psi\left(6 \phi^{\prime}+3 p \psi^{\prime}+4 \psi^{\prime \prime}\right)-2 q \psi^{2}-8 \psi^{\prime 2}\right)\right)=0,
\end{aligned}
$$

then conditions

$$
p_{1}(z)=p(z), q_{1}(z)=q(z), r_{1}(z)=r(z), s_{1}(z)=s(z)
$$

and

hold.

$$
\phi=p r-16 s+4 r^{\prime}, \quad \psi=p q-6 r+4 q^{\prime}
$$

Proof. We substitute $w(z)=(S \xi)(z)$ into equation (2.4) with unknown coefficients and then replace $\xi$ by the ratio of $y$ and $y_{1}$. Replacing the fourth and higher order derivatives of $y$ and $y_{1}$ by using the linear equations, we collect the coefficients of $y$, $y_{1}$ and their derivatives up to order 3 . In the result we obtain a system of equations on the coefficients of linear and nonlinear equations, from which we get the desired result.

Theorem 2. Let $y$ be a solution of equation (2.1) and $y_{1}$ be a solution of the third order linear differential equation of the form

$$
y^{\prime \prime \prime}(z)+q_{1}(z) y^{\prime \prime}(z)+r_{1}(z) y^{\prime}(z)+s_{1}(z) y(z)=0 .
$$

If the function $w(z)=(S \xi)(z)$ with $\xi=y / y_{1}$ solves the nonlinear differential equation (2.4), then we have conditions (2.5) for (2.4) and three additional conditions on the coefficients of the linear equation (2.6)

$$
q_{1}^{\prime}=q_{1}^{2}+q-p q_{1}-r_{1}, r_{1}^{\prime}=r-p r_{1}+q_{1} r_{1}-s_{1}, s_{1}^{\prime}=s_{1}\left(q_{1}-p\right)+s .
$$

Proof. We substitute $w(z)=(S \xi)(z)$ into equation (2.4), (2.5) with unknown coefficients and then replace $\xi$ by the ratio of $y$ and $y_{1}$. Replacing the fourth and higher order derivatives of $y$ and the third and higher order derivatives of $y_{1}$ by using the linear equations, we collect the coefficients of $y, y_{1}$ and their derivatives up to order 
3 , order 2 and order 1 respectively. In the result we obtain a system of equations on the coefficients of linear and nonlinear equations, from which we get the desired result.

Example 1. Assume that we know a particular solution $y$ and coefficient $p$ of equation $(2.1)$

$$
y(z)=z, p(z)=b,
$$

where $b$ is a constant. We substitute functions (2.8) into equations (2.1), (2.2). Solving the obtained equations we find

$$
q(z)=\frac{3 b^{2}}{8}, s(z)=-\frac{r(z)}{z} .
$$

Assume that we know a particular solution $y_{1}$ and coefficient $q_{1}$ of the equation (2.6)

$$
y_{1}(z)=z, q_{1}(z)=b .
$$

Then from equation (2.6) we find

$$
s_{1}(z)=-\frac{r_{1}(z)}{z} .
$$

We substitute functions (2.8) -(2.11) into equations (2.7) . Solving the resulting equations we find

$$
r_{1}(z)=\frac{3 b^{2}}{8}, r(z)=-\frac{3 b^{2}}{8 z} .
$$

We substitute functions (2.10), (2.11) into equation (2.6) and obtain

$$
8 z y^{\prime \prime \prime}+8 b z y^{\prime \prime}+3 b^{2} z y^{\prime}-3 b^{2} y=0 .
$$

The substitution

$$
y=z \int v(z) d z
$$

reduces equation (2.13) to the second order differential equation

$$
8 z v^{\prime \prime}+8(b z+3) v^{\prime}+b(3 b z+16) v=0 .
$$

The general solution of equation (2.15) is of the form

$$
v=e^{-\frac{1}{4} i(\sqrt{2}-2 i) b z}\left(C_{1} U_{1}+C_{2} L_{-\frac{3}{2}-\frac{i}{\sqrt{2}}}^{2}\left(\frac{b i z}{\sqrt{2}}\right)\right),
$$

where $U_{1}=U\left(\frac{3}{2}+\frac{i}{\sqrt{2}}, 3, \frac{b i z}{\sqrt{2}}\right)$ is the confluent hypergeometric function and has the integral representation

$$
U(a, b, z)=\frac{1}{\Gamma(a)} \int_{0}^{\infty} e^{-z t} t^{a-1}(1+t)^{b-a-1} d t ; L_{-\frac{3}{2}-\frac{i}{\sqrt{2}}}^{2}\left(\frac{b i z}{\sqrt{2}}\right)
$$

is the Laguerre polynomial, that satisfy the differential equation

$$
z v^{\prime \prime}+(3-z) v^{\prime}-\left(\frac{3}{2}+\frac{i}{\sqrt{2}}\right) v=0,
$$


and $C_{1}, C_{2}$ are arbitrary constants.

The general solution of equation (2.13) is of the form (2.14), (2.16). We choose, for example, the values of the arbitrary constants equal to $C_{1}=1, C_{2}=0$. Then the particular solution is

$$
y_{1}=z \int e^{-\frac{1}{4} i(\sqrt{2}-2 i) b z} U\left(\frac{3}{2}+\frac{i}{\sqrt{2}}, 3, \frac{b i z}{\sqrt{2}}\right) d z
$$

and

Then

$$
\xi=\left(\int e^{-\frac{1}{4} i(\sqrt{2}-2 i) b z} U\left(\frac{3}{2}+\frac{i}{\sqrt{2}}, 3, \frac{b i z}{\sqrt{2}}\right) d z\right)^{-1} .
$$

$$
\begin{aligned}
w & =\frac{\xi^{\prime \prime \prime}}{\xi^{\prime}}-\frac{3}{2}\left(\frac{\xi^{\prime \prime}}{\xi^{\prime}}\right)^{2} \\
& \left.=\frac{b^{2}}{16}\left(U^{-2}\left(3(7+6 \sqrt{2} i) U_{2}^{2}+2 U_{1}(5-2 i \sqrt{2}) U_{2}-(13+8 i \sqrt{2}) U_{3}\right)\right)-2 i \sqrt{2}-1\right),
\end{aligned}
$$

where

$$
U_{2}=U\left(\frac{5}{2}+\frac{i}{\sqrt{2}}, 4, \frac{b i z}{\sqrt{2}}\right), U_{3}=U\left(\frac{7}{2}+\frac{i}{\sqrt{2}}, 5, \frac{b i z}{\sqrt{2}}\right) .
$$

The differential equation (2.4), (2.5) has coefficients (2.8), (2.9), (2.12) and

$$
\phi(z)=-\frac{3 b^{2}(12+b z)}{8 z^{2}}, \psi(z)=\frac{3 b^{2}(6+b z)}{8 z} .
$$

Then according to Theorem 2 this nonlinear differential equation has a solution (2.17) which can be easily verified by substitution.

Theorem 3. Let y be a general solution of the third order linear differential equation (2.6), (2.7). Then this solution is a three parameter family of solutions of the fourth order linear differential equation (2.1), (2.2).

Proof. The proof is computational.

Theorem 4. Let y be a solution of equation (2.1) and $y_{1}$ be a solution of the second order linear differential equation of the form

$$
y^{\prime \prime}(z)+r_{1}(z) y^{\prime}(z)+s_{1}(z) y(z)=0 .
$$

If the function $w(z)=(S \xi)(z)$ with $\xi=y / y_{1}$ solves a nonlinear differential equation (2.4), then we have condition (2.5) for (2.4) and two additional conditions on the coefficients of the linear equation (2.18)

$$
\begin{aligned}
& r_{1}^{\prime \prime}=-p r_{1}^{\prime}+p r_{1}^{2}-p s_{1}-q r_{1}+r+3 r_{1} r_{1}^{\prime}+2 r_{1} s_{1}-r_{1}^{3}-2 s_{1}^{\prime}, \\
& s_{1}^{\prime \prime}=p r_{1} s_{1}-p s_{1}^{\prime}-q s_{1}+2 s_{1} r_{1}^{\prime}+r_{1} s_{1}^{\prime}-r_{1}^{2} s_{1}+s+s_{1}^{2} .
\end{aligned}
$$


Proof. We substitute $w(z)=(S \xi)(z)$ into equation (2.4), (2.5) with unknown coefficients and then replace $\xi$ by the ratio of $y$ and $y_{1}$. Replacing the fourth and higher order derivatives of $y$ and the second and higher order derivatives of $y_{1}$ by using the linear equations, we collect the coefficients of $y, y_{1}$ and their derivatives up to order 3 and order 1 respectively. In the result we obtain a system of equations on the coefficients of linear and nonlinear equations, from which we get the desired result.

Example 2. Let

$$
y(z)=\sqrt{z}, \quad p(z)=\frac{2}{z}, r(z)=\frac{3}{2 z^{3}} .
$$

We substitute functions (2.20) into equations (2.1), (2.2). Solving the resulting equations we find

$$
q(z)=-\frac{3}{2 z^{2}}, s(z)=-\frac{15}{16 z^{4}} .
$$

Let

$$
r_{1}(z)=-\frac{3}{z} \text {. }
$$

We substitute the relations (2.20)-(2.22) into (2.19). After simplifications we obtain the system

$$
s_{1}^{\prime \prime}(z)=s_{1}^{2}(z)-\frac{5}{z} s_{1}^{\prime}(z)-\frac{15}{2 z^{2}} s_{1}(z)-\frac{5}{16 z^{4}}, 4 z^{3} s_{1}^{\prime}(z)+16 z^{2} s_{1}(z)=30 .
$$

We find the following solution of system (2.23):

$$
s_{1}(z)=\frac{15}{4 z^{2}} \text {. }
$$

We substitute the relations (2.22), (2.24) into equation (2.18) and integrate it. We write the general solution in the form

$$
y_{1}=C_{1} z^{3 / 2}+C_{2} z^{5 / 2},
$$

where $C_{1}, C_{2}$ are arbitrary constants. We choose, for example, the values of arbitrary constants equal to one. Then we obtain $y_{1}=z^{3 / 2}+z^{5 / 2}$ and $\xi=\frac{1}{z^{2}+z}$. Then we find the solution

$$
w=\frac{\xi^{\prime \prime \prime}}{\xi^{\prime}}-\frac{3}{2}\left(\frac{\xi^{\prime \prime}}{\xi^{\prime}}\right)^{2}=-\frac{6}{(2 z+1)^{2}} .
$$

Differential equation (2.4), (2.5) for coefficients (2.20), (2.21) has the form

$$
\begin{gathered}
20\left(8 w^{3}+12 w w^{\prime \prime}-15 w^{\prime 2}\right) w^{\prime \prime \prime \prime}-280 w w^{\prime \prime \prime}-280 w^{\prime}\left(4 w^{2}-3 w^{\prime \prime}\right) w^{\prime \prime \prime}-504 w^{\prime \prime 3} \\
+192 w^{2} w^{\prime \prime 2}+8\left(56 w^{4}+255 w w^{\prime 2}\right) w^{\prime \prime}-1275 w^{4^{4}}-560 w^{3} w^{\prime 2}+64 w^{6}=0 .
\end{gathered}
$$

According to Theorem 4 equation (2.27) has the solution (2.26) that can be easily verified by the direct substitution. 
If we choose the function (2.25), then the corresponding solution of the equation (2.27) takes the form

$$
w=-\frac{6 C_{2}^{2}}{\left(2 C_{2} z+C_{1}\right)^{2}} .
$$

It is a one-parameter family of solutions. This is easily seen by introducing the substitution $c=C_{2} / C_{1}$.

Theorem 5. Let $y$ be a general solution of the second order linear differential equation (2.18), (2.19). Then this solution is a two parameter family of solutions of the fourth order linear differential equation (2.1), (2.2).

Proof. The proof is computational.

\section{CONCLUSIONS}

One research direction is to replace linear differential equations with nonlinear equations of second and higher order and to consider the Schwarzian derivative of the ratio of 2 solutions. This might give a new insight into the theory of some nonlinear special functions.

Taking into account the obtained results for the known solutions of the fourth and the second order linear equations, we can formulate the corresponding theorems for the known solutions of the fourth order linear equation and the Riccati equation, to which the second order linear equation reduces. Here it seems appropriate to use the results of [9] and the method of V. Orlov [13,14] for the study of the Riccati equation and nonlinear differential equations of the second order.

From the point of view of programming algorithms for solving the considered problems, the opportunities of Wolfram Research technologies described in [15] are essential. They significantly complement the set of tools for creating, maintaining and distributing dynamic content when constructing and studying solutions of differential equations.

\section{REFERENCES}

[1] L. Ahlfors, Mobius transformations in several dimensions. Minneapolis: Lecture notes at the University of Minnesota, 1981.

[2] A. Chichurin, "The computer method of construction of the general solution of the nonlinear differential equation of the fourth order." Studia i Materialy EWSIE, vol. 7, pp. 39-47, 2014.

[3] A. Chichurin, Recent Developments in Mathematics and Informatics, Contemporary Mathematics and Computer Science. Lublin: KUL, 2016, vol. 1, ch. 2, pp. 19-36.

[4] A. Chichurin and G. Stepaniuk, " The computer method of construction of the general solution of the linear differential equation of the third order." Studia i Materialy EWSIE, vol. 8, pp. 17-27, 2014.

[5] A. Chichurin and G. Stepaniuk, "General solution to the fourth order linear differential equation with coefficients satisfying the system of three the first order differential equations." Bulletin of Taras Shevchenko National University of Kyiv, Series: Physics and Mathematics., no. 31, pp. 7-10, 2014. 
[6] A. V. Chichurin, The Chazy equation and linear equations of the Fuchs class. Moscow: RUDN, 2003, (in Russian).

[7] A. Chichurin and G. Filipuk, "The properties of certain linear and nonlinear differential equations of the fourth order." preprint, 2019.

[8] V. Dobrovolsky, Essays on the development of the analytic theory of differential equations. Kiev: Vishcha shkola, 1974, (in Russian).

[9] G. Filipuk and A. Chichurin, Advances in Mechanics and Mathematics. Cham: Springer, 2019, vol. 41, ch. The Properties of Certain Linear and Nonlinear Differential Equations, pp. 193-200, doi: 10.1007/978-3-030-02487-1-11.

[10] N. Lukashevich, "On third-order linear equations." Differ. Equ., vol. 35, no. 10, pp. 1384-1390, 1999.

[11] N. Lukashevich and A. Chichurin, Differential equations of the first order. Minsk: BSU, 1999, (in Russian).

[12] N. Lukashevich and I. Martynov, Proc. of the International Scientific Conference "Differential Equations and Their Applications". Grodno: GrSU, 1998, pp. 78-85.

[13] V. Orlov and O. Kovalchuk, "Research of one class of nonlinear differential equations of third order for mathematical modeling the complex structures." IOP Conf. Series: Materials Science and Engineering, FORM 2018, vol. 365, no. 042045, pp. 1-8, 2018, doi: 10.1088 / 1757-899X / 365/4/042045.

[14] V. N. Orlov, Approximate method for solving scalar and matrix Riccati differential equations. Cheboksary: Chuvash State Pedagogical University, 2015, (in Russian).

[15] V. Taranchuk and M. Zhuravkov, "Development of interactive teaching materials for computer mechanics.” Vest. BSU. Ser. 1, Fiz. Mat. Inform., no. 3, pp. 97-107, 2016.

Authors' addresses

Alexander Chichurin

Institute of Mathematics and Computer Science, The John Paul II Catholic University of Lublin, ul. Konstantynow 1H, 20-708 Lublin, Poland

E-mail address: achichurinegmail.com

Galina Filipuk

Faculty of Mathematics, Informatics and Mechanics, University of Warsaw, ul. Banacha 2, 02-097 Warsaw, Poland

E-mail address: filipuk@mimuw.edu.pl 